\title{
Europäische Gesundheitssysteme: Bei den Nachbarn ist alles besser, oder nicht?
}

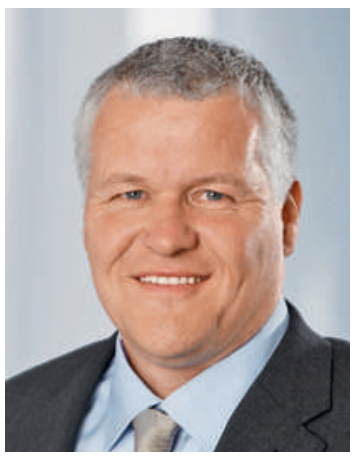

Als Einstieg wenden wir uns nach Westen und vergleichen unser Gesundheitssystem mit jenem Frankreichs. Was die Aufgabenteilung zwischen Grundversorgern und Spezialisten betrifft, sind sich Frankreich (F) und die Schweiz (CH) ähnlich: Stationäre und ambulante Leistungen werden grundsätzlich getrennt, wobei Letztere mehrheitlich durch den nicht-staatlichen ambulanten Sektor erbracht werden. Ähnliche Strukturen bestehen in Deutschland, Belgien und Österreich. 53,\% der Ärztinnen und Ärzte sind in der $\mathrm{CH}$ im ambulanten Sektor tätig, in $\mathrm{F}$ sind es 46,6\%. Ebenso ist der Anteil der gesamten Gesundheitskosten am Bruttoinlandprodukt mit $11 \%$ in der $\mathrm{CH}$ und $11,6 \%$ in $\mathrm{F}$ etwa gleich.

Wesentliche Unterschiede zeigen sich beim Zugang zur Gesundheitsversorgung: In der Schweiz werden 92\% der Patienten innerhalb eines Monats vom Spezialisten untersucht, in Frankreich sind es nur 67\%. Auch bei den Grundversorgern ist der Unterschied deutlich: in der Schweiz erfolgt die als dringlich gewünschte Konsultation in $81 \%$ der Fälle noch

\section{Ein staatliches System führt nicht zu mehr Informationsfluss zwischen Spitä- lern und nachbehandelnden Ärzten.}

am gleichen Tag, während dies in Frankreich nur in 59\% der Fälle möglich ist. Im akut medizinischen Bereich sind die Unterschiede geringer, was sich in der Letalität nach Myokardinfarkt (CH 5,9\% vs. F 6,2\%) respektive Schlaganfall (CH 7,0\% vs. F 8,5\%) zeigt. Die Schweiz hat $15,7 \%$ weniger Akut-Spitalbetten pro 100000 Einwohner (320 CH, 380 F), wobei beide Länder eine ähnliche Tendenz zu deren Reduktion zeigen.

Man sollte glauben, dass in einem relativ zentralistisch respektive staatlich (Agence régionale de la santé ARS) geregelten System der Informationsfluss zwischen Spitälern und behandelnden Ärzten deutlich besser sein sollte (Typ Netzwerk). Dies ist aber nicht der Fall, da nur 10\% der nachbehandelnden Kollegen in Frankreich innerhalb 48 Stunden eine detaillierte Information über die Entlassung der Patienten erhalten. Die Schweiz nimmt zwar keinen Spitzenplatz ein, aber immerhin ist dies in 40\% der Fall bei uns. Was die Produktivität betrifft, verwendet ein Hausarzt in der Schweiz rund 15,6 Minuten für eine Konsultation, in Frankreich dauert eine solche um 42,3\% länger mit durchschnittlich 22,2 Minuten. Erstaunlich ist, dass 91,3\% der Befragten in der Schweiz die Konsultationsdauer als genügend beurteilen, in Frankreich jedoch «nur» $85,4 \%$ der Befragten.

Interessant ist auch, dass sich die Vergütung der Leistung in Kaufkraftparität reziprok zur allgemeinen Wahrnehmung verhält: Bei Grundversorgern in Frankreich liegt diese um 23,3\% tiefer als bei ihren Kollegen aus der Schweiz. Bei Spezialisten ist es gerade umgekehrt, da liegen die Kollegen aus der Schweiz 19,4\% tiefer als in Frankreich [1].

\section{Im Patientenvergleich schneidet das Schweizer Gesundheitswesen besser ab als das französische.}

In der Schweiz sind 69\% der Bevölkerung mit dem Gesundheitssystem zufrieden, in Frankreich sind es nur 40\%. Interessant ist auch, dass in Frankreich Rechnungen unter $100 \$$ in $42,1 \%$ der Fälle aus der eigenen Tasche bezahlt werden [2], obschon alle Bürger bei der staatlichen Krankenkasse versichert sind und rund 95\% zusätzlich eine weitere Versicherung abgeschlossen haben (gemeinnützige Versicherung «Mutuelle» oder private Zusatzversicherung). Dies ist in der Schweiz «nur» in 26,8\% der Fall. Ebenso verweigern die Versicherungen in Frankreich in 17\% der Fälle die Übernahme der Kosten; die Schweiz liegt mit 15,7\% leicht tiefer.

Ein stark staatlich reguliertes System ist somit kein Garant für soziale Gerechtigkeit oder eine hohe Versicherten- bzw. Patientenzufriedenheit. Hingegen lässt sich in einem stark reglementierten Gesundheitswesen ein Rückgang der Produktivität beobachten. Im Vergleich mit Frankreich schneidet das Schweizer Gesundheitswesen somit nicht schlecht oder sogar besser ab.

\section{Dr. med. Remo Osterwalder, Mitglied des Zentralvorstandes der FMH, Departementsverantwortlicher Freipraktizierende Ärztinnen und Ärzte}

1 OECD Health Data 2008.

2 Commonwealth Fund, International Health Policy Survey 2013. 